\title{
Three-dimensional magnetic resonance flow analysis in a ventricular assist device
}

\author{
Michael Markl, PhD, ${ }^{a}$ Christoph Benk, MS, ${ }^{b}$ Dominik Klausmann, MS, ${ }^{b}$ Aurelien F. Stalder, MS, ${ }^{a}$ \\ Alex Frydrychowicz, MD, ${ }^{a}$ Jürgen Hennig, $\mathrm{PhD}^{\text {a }}$ and Friedhelm Beyersdorf, $\mathrm{MD}^{\mathrm{b}}$
}

Video clip is available online.

From the Departments of Diagnostic Radiology, Medical Physics, ${ }^{a}$ and Cardiovascular Surgery, ${ }^{\mathrm{b}}$ University Hospital Freiburg, Germany.

Sponsored by Deutsche Forschungsgemeinschaft (DFG) Grant No. MA 2383/3-1.

Received for publication Jan 11, 2007; revisions received April 13, 2007; accepted for publication May 2, 2007.

Address for reprints: Michael Markl, PhD, University Hospital Freiburg, Department of Diagnostic Radiology, Medical Physics, Hugstetter Strasse 55, 79106 Freiburg, Germany (E-mail: michael.markl@uniklinikfreiburg.de).

J Thorac Cardiovasc Surg 2007;134:1471-6

$0022-5223 / \$ 32.00$

Copyright () 2007 by The American Association for Thoracic Surgery

doi:10.1016/j.jtcvs.2007.05.024

Objective: The assessment of flow characteristics inside ventricular assist devices by magnetic resonance imaging techniques may provide insight into the mechanisms underlying the high rate of thromboembolic events after implantation of a ventricular assist device. Furthermore, these investigations may form the basis to optimize the device's design and its need for anticoagulation. The purpose of this study was to integrate a clinical routine ventricular assist device into a flow circuit with realistic geometric and pulsatile inflow conditions. Combination with flow-sensitive magnetic resonance imaging at $3 \mathrm{~T}$ permitted the detailed analysis of local and global 3-dimensional flow dynamics in a realistic environment.

Methods: A commercially available ventricular assist device was integrated into a magnetic resonance-compatible flow circuit. Flow-sensitive 3-dimensional magnetic resonance imaging was performed to measure time-resolved 3-directional flow velocities in the entire device. Advanced computer-aided 3-dimensional flow visualization methods were used to derive a comprehensive picture of flow dynamics within the ventricular assist device system.

Results: On the basis of the ventricular assist device model system, magnetic resonance imaging, and flow visualization, the first 4-dimensional functional magnetic resonance imaging analysis of flow characteristics inside an operating clinical routine ventricular assist device chamber system is reported. Detailed visualization of flow patterns and local changes in flow characteristics were successfully performed and revealed locally accelerated, vortical, and helical flow regions inside the geometry of the device.

Conclusions: Complex flow patterns such as vortex formation and locally accelerated flow demonstrate the potential of the presented method to further deepen the understanding of complex and regionally different flow characteristics inside ventricular assist devices.

$\mathrm{M}$ echanical circulatory support has evolved as a realistic therapeutic option for patients in end-stage heart failure. ${ }^{1-7}$ There has been an improvement in many aspects of the ventricular assist device (VAD) over the past decades, resulting in an increased implantation rate in many cardiac surgical centers. ${ }^{1-3,8}$ However, some potentially lethal and devastating complications remain after VAD implantation, including thrombus formation and embolization, infections, and device failures. ${ }^{9,10}$ To prevent thromboembolization, most devices need a sophisticated anticoagulation regimen that in turn creates problems of its own (eg, bleeding or stroke). ${ }^{5,6,11}$

An ideal approach to investigate blood flow in a VAD would require direct assessment of flow characteristics during the in vivo setting. A potential solution may be offered by two- or three-dimensional (2D or 3D) time-resolved flowsensitive magnetic resonance imaging (MRI) methods, which have been widely applied 


\section{Abbreviations and Acronyms \\ 2D, 3D, 4D = two-, three-, four-dimensional \\ MR $=$ magnetic resonance \\ MRI = magnetic resonance imaging \\ VAD = ventricular assist device}

for the qualitative and quantitative assessment of in vivo blood flow. $^{12-14}$ More recently, true 3D time-resolved MRI with 3-directional flow encoding (flow sensitive 4D-MRI) has been introduced. ${ }^{15,16}$ Such techniques permit the detailed analysis of dynamic blood flow characteristics within an acquired $3 \mathrm{D}$ data volume covering the entire thoracic aorta, ${ }^{17-19}$ the heart, ${ }^{20}$ or the peripheral arteries. ${ }^{21}$

However, implanted VADs are often not MR compatible or the patient setup does not allow MR acquisitions, and in vivo flow studies thus cannot be performed directly. For the understanding of flow characteristics within VADs to be enhanced, it is therefore of great interest to provide a realistic in vitro VAD model system. ${ }^{22}$ The experimental simulation and analysis of 3D flow patterns in such a system may have the potential to deepen the understanding of the development of flow patterns and evaluate functional consequences such as thrombus development.

To our knowledge, no detailed 3D flow analysis of VAD flow characteristics has been presented to date, and the actual flow patterns inside VADs in pulsatile flow mode are generally unknown. The overall aim of this study was to test the feasibility of an MR-compatible VAD model system for the assessment of regional 3D flow characteristics within the device. Thus, it was the purpose of this study to perform flow-sensitive 4D MRI measurements in combination with detailed 3D visualization for the assessment of 3D flow dynamics inside a VAD model system. Specifically, the inflow waveform at the inlet of the vascular model was adjusted to match the blood flow characteristics typically used in vivo by using an identical control and pump unit.

As a long-term objective, such model systems could also be used to optimize the design of VADs and to test different inflow conditions and their effects on the blood flow characteristics and VAD performance.

\section{Materials and Methods \\ VAD Model System}

A commercially available VAD (MEDOS VAD-Pump-Chamber; MEDOS Medizintechnik AG, Stolberg, Germany) was integrated into a closed MR-compatible flow circuit including polyvinyl chloride tubes, a reservoir, and an air pressure pump and control unit, which was placed outside the scanner room.

The VAD (chamber size $=60 \mathrm{~mL}$ ) consisted of a pneumatically driven blood pump that may serve as support of the left or right ventricle as well as biventricular assistance for both ventricles. The pulsatile volume flow created by the control and pump unit was adjusted by two opposite permeable bulbs at the inlet or outlet side with inserted 3-leaflet valves similar to the natural aortic valve. The pressure wave was transmitted to the pump chamber by a double-layered membrane transferring the supplied periodic pressure changes into pulsatile blood flow through the VAD.

The pump and control unit consisted of a clinical routine device (MEDOS VAD-Driving Unit; MEDOS Medizintechnik AG) used for in vivo VAD operation, which was modified by use of long tubes (approximately $8 \mathrm{~m}$ ) to connect the pump unit outside the MR room to the VAD circuit inside the MR scanner. Periodic pressure waveforms were transferred to the VAD circuit to generate pulsatile flow (systolic pressure $=180 \mathrm{~mm} \mathrm{Hg}$, diastolic pressure $=-20 \mathrm{~mm} \mathrm{Hg}$, systolic fraction $=35 \%$ of flow cycle) within the VAD model system.

A reservoir with approximately $5 \mathrm{~L}$ of additional fluid was integrated into the flow circuit to simulate in vivo pressure conditions.

The fluid (water) was doped with a gadolinium-based contrast agent (Magnevist; gadopentetate dimeglumine, Schering, Germany) to maximize signal-to-noise ratio. A schematic illustration of the experimental setup is provided in Figure 1.

\section{MRI Measurements}

All MR experiments were performed with a 3-T MRI system (Magnetom TRIO; Siemens, Germany) with a standard 8-channel body coil. MR flow measurements covering the entire VAD were performed by time-resolved 3D phase contrast MRI (flow-sensitive 4D MRI) with interleaved 3-directional velocity encoding (spatial resolution $=2.0 \times 1.9 \times 2.0 \mathrm{~mm}^{3}$, flip angle $=15^{\circ}$, echo time $/$ repetition time $=3.7 / 6.1 \mathrm{~ms}$, velocity sensitivity $=150 \mathrm{~cm} / \mathrm{s}){ }^{23}$ Measurements were prospectively gated to the RR interval simulated by the pump system, and 14 time frames with a temporal resolution of $48.8 \mathrm{~ms}$ were collected within the flow cycle.

\section{Data Analysis-3D Flow Visualization}

After noise masking and eddy current correction, ${ }^{24}$ the measured velocity data were loaded into a commercially available software package (EnSight; CEI, Apex, NC) for 3D visualization of the flow characteristics inside the VAD system. The software permitted interactive $3 \mathrm{D}$ data navigation, definition of cut planes transecting the 3D data volume at any user-selected angle, and 3D visualization of the measured flow velocities as vector graphs and 3D streamlines. ${ }^{25}$

Vector graphs were generated on predefined 2D cut planes and illustrated the direction and magnitude of the measured flow velocities for different frames within the flow cycle.

The 3D streamlines represent lines locally parallel to the 3directional flow velocity field for a given time-frame within the pulsatile flow cycle. All tracings were color-coded according to the measured local low velocities and originated from a set of equidistant grid points on an emitter plane, which was interactively positioned within the $3 \mathrm{D}$ imaging volume.

\section{Results}

Time-resolved 3D VAD Geometry

To analyze the performance and associated geometric changes of the VAD during periodic pressure variation, we 


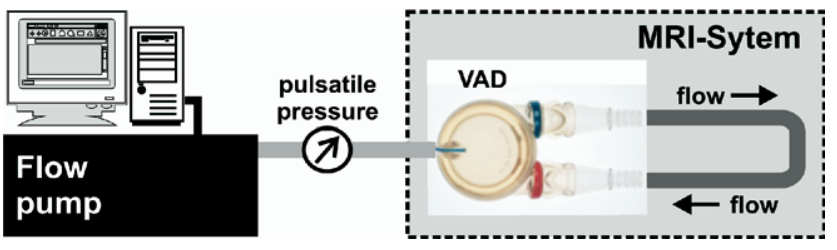

Figure 1. Schematic illustration of the setup used for MRI-based 3D flow analysis. A commercially available VAD was integrated into a closed flow circuit and connected to a control and pump unit. Periodic pressure variation identical to in vivo VAD operation was used to generate pulsatile flow within the VAD model system. Additional realistic boundary conditions were achieved by adding a reservoir to the circuit (not shown) to reach a total fluid volume of approximately $5 \mathrm{~L}$, similar to the typical blood volume of a human subject. VAD, Ventricular assist device; MRI, magnetic resonance imaging.

interactively positioned $2 \mathrm{D}$ cut planes in the time-resolved 3D magnitude data set. Figure 2, $A$ shows the state of the VAD during filling, inflow, and mostly homogenous signal inside maximally expanded VAD chamber volume. Figure $2, B$ represents the state of the VAD during maximum pressure, outflow, and reduced signal inside the maximally compressed VAD chamber volume. Note that metallic components at the inlet and outlet result in signal loss (open arrows). The cyclic volume changes during the entire flow cycle can be best appreciated in the supplemental movie file.

\section{D VAD Flow Analysis}

To depict the 3D flow characteristics within the entire VAD for the whole flow cycle, we applied 3D streamline visualization. Emitter planes normal to the VAD lumen were placed near the inlet and outlet of the VAD chamber.

The results for all 14 analyzed time frames within the flow cycle are shown in Figure 3. Similar to Figure 2, the magnitude data additionally reflect the state of the VAD chamber during the flow cycle. Higher signal intensities in the VAD chamber in frames 6 to 10 are consistent with minimal pressure and expanded VAD chamber volume.

The 3D streamlines demonstrated complex flow patterns ranging from flow channels along the VAD border during filling (frames 1-5) followed by circular flow patterns and vortex formation in the VAD lumen (frames 6-10). Accelerated flow before and during filling of the VAD lumen in frames 2 to 6 is followed by high outflow during phases 10 to 14 . Note that flow channels along the borders of the VAD volume persist throughout the entire flow cycle.

A more detailed visualization of 3D flow patterns for two selected time frames is shown in Figure 4. During filling of the VAD chamber (Figure 4, A), high and accelerated flow at the inlet can clearly be appreciated. Moreover, the existence of a considerable flow vortex inside the expanded VAD chamber is clearly visible (white arrows).
The 3D flow characteristics at the onset of the filling phase are illustrated in Figure 4, B. Although the VAD chamber is still almost fully compressed, a small flow channel (yellow arrows) along the outer rim of the VAD chamber has already developed.

Similar flow patterns were detected by vector graph visualization as depicted in Figure 5 for a time frame corresponding to the filling phase in Figure 4, A. Considerable vortex formation in the central VAD lumen (yellow arrows) as well as accelerated flow at the inlet (white arrow) are clearly visible.

A: min pressure, VAD filling

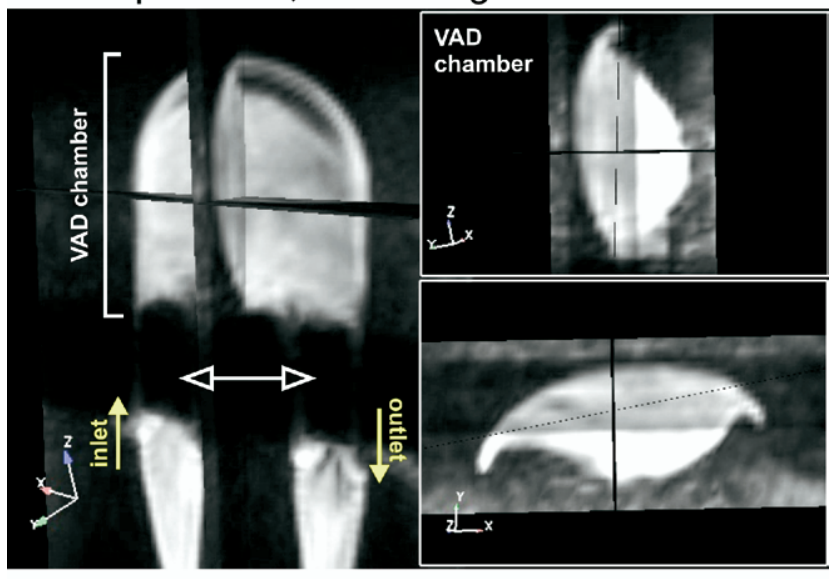

\section{B: max. pressure, VAD out-flow}

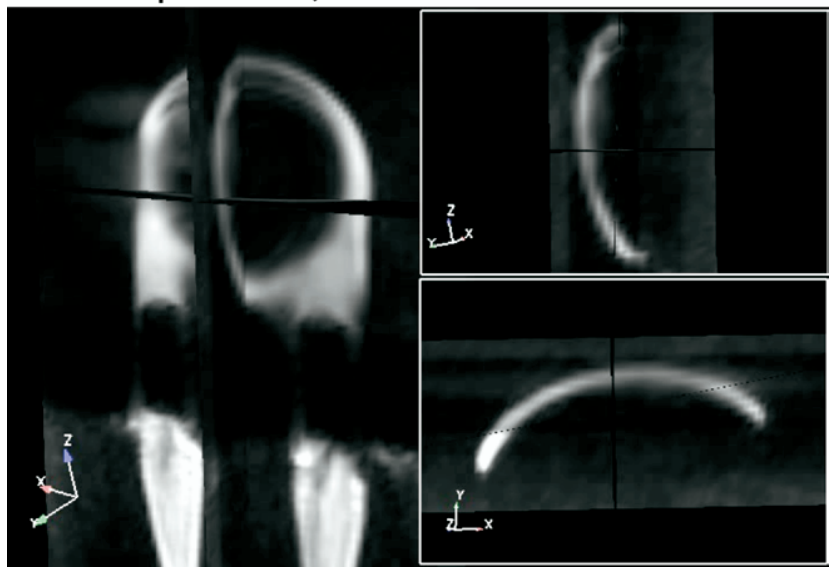

Figure 2. Time-resolved 3D MRI in 3 orthogonal cut planes transecting the VAD fluid volume. Different time frames within the pulsatile pressure-driven flow cycle clearly show expanded VAD chamber volume during filling (top, A) and maximum volume compression during maximum pressure and outflow (bottom, B). Note that metal artifacts near the VAD valves at the inlet and outlet results in signal void in these regions (open arrows). See also supplemental video. VAD, Ventricular assist device. 

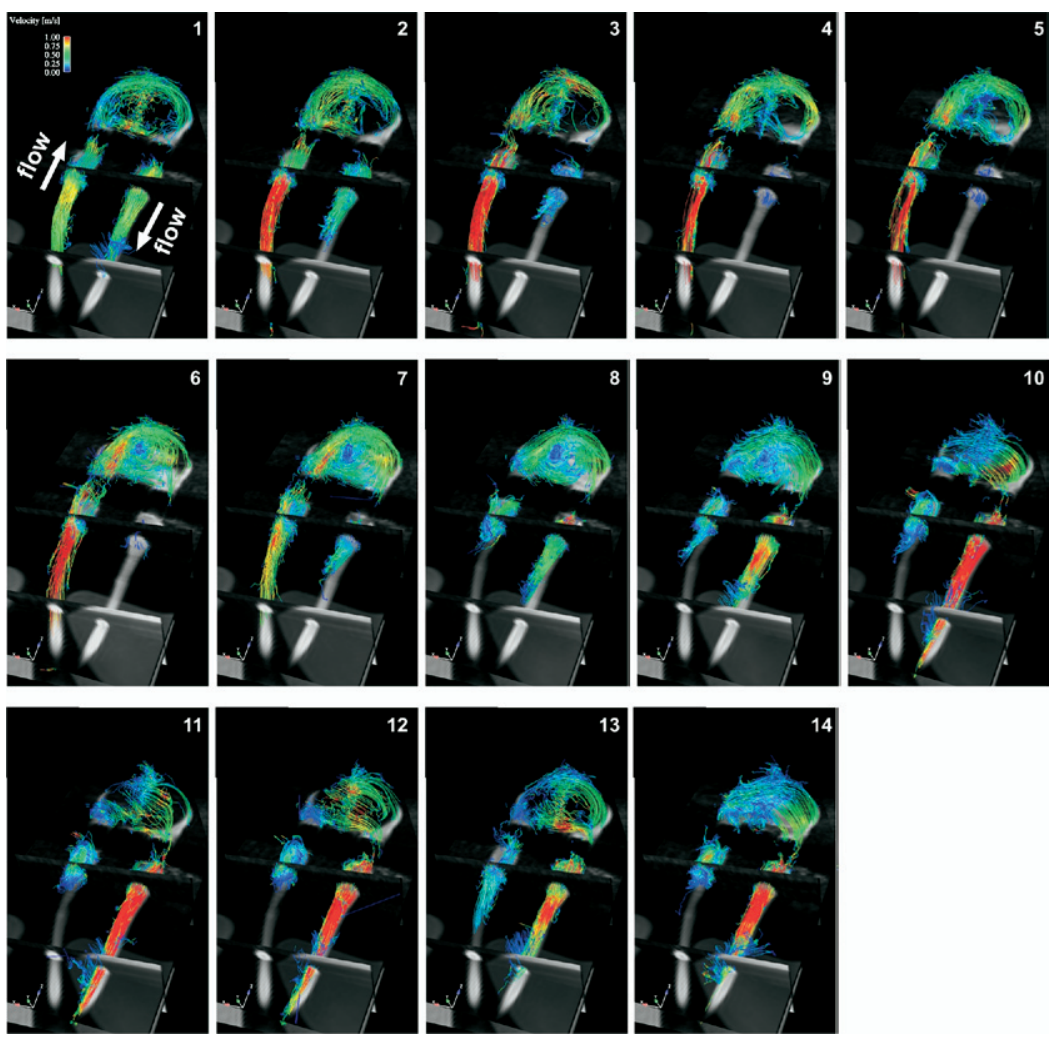

\section{A: VAD filling}

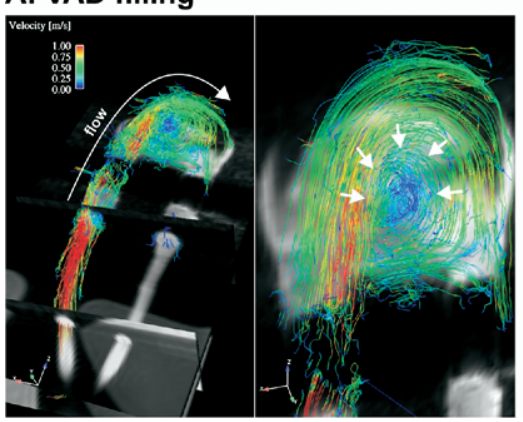

\section{B: VAD in- and out-flow}

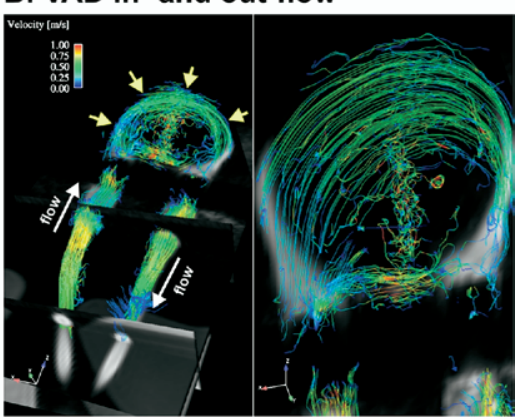

Figure 3. 3D streamline visualization of the measured flow characteristics in the entire VAD throughout the experimentally simulated pulsatile flow cycle. Overall flow directions at the inlet (left arrow) and outlet (right arrow) of the VAD chamber are indicated in the top left images. A more detailed visualization of local flow characteristics for frames 1 and 6 is provided in Figure 4.

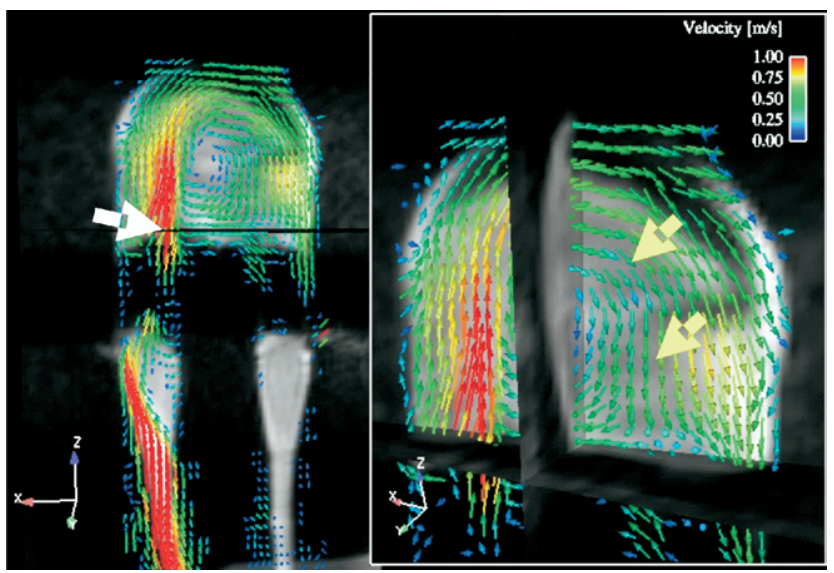

Figure 5. Detailed 3D flow characteristics during the filling phase of the VAD. Visualization of the measured flow velocities was performed as 3-directional vector graphs in a plane transecting the VAD volume as well as inflow and outflow channels. Large white and yellow arrows indicate regions of flow acceleration and vortex formation, respectively. 


\section{Discussion}

Results of this study indicate that in vitro flow experiments could successfully be performed inside a VAD model system. The integration into an optimized pulsatile flow circuit in combination with the application of flow-sensitive 4D MRI permitted detailed analysis of dynamic flow characteristics in a realistic environment resembling in vivo conditions.

Flow-sensitive 4D MRI may thus have the potential to enhance the understanding of flow characteristics inside VAD systems with different size, geometry, and inflow conditions (eg, nonpulsatile versus pulsatile flow). Moreover, the detailed visualization flow patterns and/or local changes in flow velocities may help to identify vortical, helical flow regions inside the VAD geometry that are likely to develop thrombi or other functional deficits that may impair VAD performance.

It has to be noted, however, that thrombosis as a result of vortical or helical flow was not directly observed in this study. In our evaluation of the observed flow patterns inside the VAD, we hypothesize that such flow features may lead to thrombosis as described in the literature for similar in vivo flow patterns. ${ }^{26-30}$ Future studies should therefore include a more detailed analysis of parameters directly related to thrombogenesis, such as changes in wall shear forces that can be derived from the measured flow velocity vector fields. ${ }^{27,30}$

In addition, VAD model systems and experimental MR flow simulations in a controlled environment offer the opportunity to modify location, size, and shape of the VAD and optimize its performance with respect to generation of target flow characteristics. Moreover, MR flow analysis coupled with realistic VAD model systems has the potential to permit the detailed analysis of the influence of size and shape of different VAD geometries on local flow dynamics, for example, change in flow vortex formation as a function of VAD chamber size and shape. Larger studies may thus provide new information for the optimized design of VAD geometry and/or inflow conditions.

Limitations of this study include the use of water for all flow experiments. A more realistic experimental setup would require the use of human plasma or blood in combination with appropriate temperature control. Future studies using blood mimicking fluids or even real plasma or blood are thus warranted to analyze the influence of viscosity and density on flow dynamics inside VAD systems.

In addition, the inflow boundary characteristics may have been altered compared with in vivo conditions owing to the long tubes that were necessary to deliver the periodic pressure changes to the VAD system. Further studies are thus planned to understand the waveform variation of the propagating wave to permit a more precise generation of realistic inflow conditions.
The VAD used for this study demonstrated metal artifacts, for example, signal loss, in regions at the inlet and outlet.

The existence of such metal components limits the application of sensitive MRI for the assessment of flow characteristics inside all regions of the VAD. Therefore, no results could be obtained concerning the flow characteristics in the "sinus of Valsalva-type" recesses of the inflow and outflow cannulas that are originally designed to resemble nature in that respect. Clinically, these sinuses are frequently areas of thrombus formations (especially on the right side), leading eventually to the exchange of the pump chamber.

Nevertheless, metal components of the investigated VAD only partially obstructed the device, and flow patterns were successfully analyzed in large sections of the VAD, such as the pump chamber as well as the inlet and outlet. The metal components, which are used to support the artificial valve systems in the VAD, consist of simple rings mounted at the outer layer of the VAD at the level of the valve base. These can be removed and replaced by nonmetallic materials without any additional changes or modifications of the VAD chamber, valves, and flow channels. It is thus expected that the modified nonmetallic VAD will provide flow patterns identical to those of the original device. Future studies will therefore be performed with completely MR-compatible model systems that will provide insights into 3D flow characteristics within all regions of the VAD including flow patterns through and near the valve systems.

Once such optimized model systems are established, incremental changes in VAD design (eg, chamber geometry or valve design) and/or boundary conditions (eg, inflow pressures) can be used to systematically evaluate and optimize VAD operation by minimizing the extent of recirculation zones and vertical flow fields.

Although the MRI flow characterization reported in this article has not been compared with other flow analysis methods, additional measurement techniques or computational methods exist that have been used to assess flow within model systems. ${ }^{30-35}$

In addition to optical measurements (particle trace velocimetry $)^{30,31}$ or ultrasound using gas bubbles, ${ }^{32}$ many studies using computational fluid dynamics ${ }^{33-35}$ have been performed. Analysis was typically based on model geometries or realistic vascular computer representations derived from angiography data. However, to our knowledge, no detailed analysis of 3D flow dynamics using full VAD coverage has been reported on the basis of these modalities. Specifically, no computational fluid dynamics study regarding flow patterns inside a VAD using realistic boundary conditions (ie, inflow waveforms and elasticity of VAD chamber) has been reported to date. Owing to the complexity of modeling realistic pump chamber performance using 
computer modeling, MRI may provide an advantage over computational fluid dynamics because the real VAD in combination with pump and control unit can be directly integrated into the 3D flow analysis process.

The methods used in this pilot study permitted the detailed evaluation of the 3D evolution of local flow patterns inside a routine clinical VAD. Results illustrate that flowsensitive 4D MRI could successfully be used to analyze local and global flow dynamics in a realistic VAD model system.

\section{References}

1. McMurray JJ, Pfeffer MA. Heart failure. Lancet. 2005;365:1877-89.

2. DeBakey ME. Development of mechanical heart devices. Ann Thorac Surg. 2005;79:S2228-31.

3. Collard E, Van Dyck MJ, Jacquet LM. Ventricular assist devices. Curr Opin Anaesthesiol. 2003;16:33-43.

4. Siegenthaler MP, Frazier OH, Beyersdorf F, Martin J, Laks H, Elefterides J, et al. Mechanical reliability of the Jarvik 2000 heart. Ann Thorac Surg. 2006;81:1752-9.

5. Siegenthaler MP, Westaby S, Frazier OH, Martin J, Banning A, Robson D, et al. Advanced heart failure: feasibility study of long-term continuous axial flow pump support. Eur Heart J. 2005:26:1031-8.

6. Siegenthaler MP, Martin J, van de Loo A, Doenst T, Bothe W, Beyersdorf F. Implantation of the permanent Jarvik 2000 left ventricular assist device. J Am Coll Cardiol. 2002;39:1764-72.

7. Siegenthaler MP, Brehm K, Strecker T, Hanke T, Nötzold A, Olschewski M, et al. The Impella Recover microaxial left ventricular assist device reduces mortality for postcardiotomy failure: a threecenter experience. J Thorac Cardiovasc Surg. 2004;127:812-22.

8. Ohuchi K, Takatani S. Currently available ventricular-assist devices: capabilities, limitations and future perspectives. Expert Rev Med Devices. 2006;3:195-205.

9. Martin J, Friesewinkel O, Benk C, Sorg S, Schultz SD, Beyersdorf F. Improved durability of the HeartMate XVE left ventricular assist device provides safe mechanical support up to 1 year but is associated with high risk of device failure in the second year. J Heart Lung Transplant. 2006;25:384-90.

10. Gordon RJ, Quagliarello B, Lowy FD. Ventricular assist devicerelated infections. Lancet Infect Dis. 2006;6:426-37.

11. Martin J, Siegenthaler MP, Friesewinkel O, Fader T, van de Loo A, Trummer G, et al. Implantable left ventricular assist device for treatment of pulmonary hypertension in candidates for orthotopic heart transplantation-a preliminary study. Eur J Cardiothorac Surg. 2004; 25:971-7.

12. Nayler GL, Firmin DN, Longmore DB. Blood flow imaging by cine magnetic resonance. J Comput Assist Tomogr. 1986;10:715-22.

13. Firmin DN, Nayler GL, Klipstein RH, Underwood SR, Rees RS, Longmore DB. In vivo validation of MR velocity imaging. J Comput Assist Tomogr. 1987;11:751-6.

14. Pelc NJ, Herfkens RJ, Shimakawa A, Enzmann DR. Phase contrast cine magnetic resonance imaging. Magn Reson Q. 1991;7:229-54.

15. Wigstrom L, Sjoqvist L, Wranne B. Temporally resolved 3D phasecontrast imaging. Magn Reson Med. 1996;36:800-3.

16. Markl M, Chan FP, Alley MT, Wedding KL, Draney MT, Elkins CJ, et al. Time-resolved three-dimensional phase-contrast MRI. J Magn Reson Imaging. 2003;17:499-506.
17. Bogren HG, Buonocore MH. 4D magnetic resonance velocity mapping of blood flow patterns in the aorta in young vs. elderly normal subjects. J Magn Reson Imaging. 1999;10:861-9.

18. Markl M, Draney MT, Miller DC, Levin JM, Williamson EE, Pelc NJ, et al. Time-resolved three-dimensional magnetic resonance velocity mapping of aortic flow in healthy volunteers and patients after valvesparing aortic root replacement. J Thorac Cardiovasc Surg. 2005;130: $456-63$.

19. Kvitting JP, Ebbers T, Wigstrom L, Engvall J, Olin CL, Bolger AF. Flow patterns in the aortic root and the aorta studied with timeresolved, 3-dimensional, phase-contrast magnetic resonance imaging: implications for aortic valve-sparing surgery. $J$ Thorac Cardiovasc Surg. 2004;127:1602-7.

20. Kilner PJ, Yang GZ, Wilkes AJ, Mohiaddin RH, Firmin DN, Yacoub MH. Asymmetric redirection of flow through the heart. Nature. 2000; 404:759-61.

21. Frydrychowicz A, Winterer J, Zaitsev M, Jung B, Hennig J, Langer M, et al. Visualization of iliac and proximal femoral artery hemodynamics using time-resolved 3D phase contrast MRI at 3T. J Magn Reson Imaging. 2007;25:1085-92.

22. Carroll FE Jr, Parker RE, Loyd JE, Holburn GE, King JC, Roos CF, et al. Inexpensive, air-driven ventricular assist or replacement device for use in MR research. Invest Radiol. 1990;25:579-82.

23. Markl M, Harloff A, Bley T, Zaitsev M, Jung B, Weigang E, et al. Time resolved 3D MR velocity mapping at 3T: improved navigator gated assessment of vascular anatomy and blood flow. J Magn Reson Imaging. 2007;25:824-31.

24. Walker PG, Cranney GB, Scheidegger MB, Waseleski G, Pohost GM, Yoganathan AP. Semiautomated method for noise reduction and background phase error correction in MR phase velocity data. $J$ Magn Reson Imaging. 1993;3:521-30.

25. Buonocore MH. Visualizing blood flow patterns using streamlines, arrows, and particle paths. Magn Reson Med. 1998;40:210-26.

26. Wurzinger LJ, Blasberg P, Schmid-Schonbein H. Towards a concept of thrombosis in accelerated flow: rheology, fluid dynamics, and biochemistry. Biorheology. 1985;22:437-50.

27. Sato M, Ohshima N. Effect of wall shear rate on thrombogenesis in microvessels of the rat mesentery. Circ Res. 1990;66:941-9.

28. Weiss HJ. Flow-related platelet deposition on subendothelium. Thromb Haemost. 1995;74:117-22.

29. Mandrusov E, Puszkin E, Vroman L, Leonard EF. Separated flows in artificial organs. A cause of early thrombogenesis? ASAIO J. 1996;42: M506-13.

30. Mussivand T, Day KD, Naber BC. Fluid dynamic optimization of a ventricular assist device using particle image velocimetry. ASAIO J. 1999;45:25-31.

31. Yamane T, Miyamoto Y, Tajima K, Yamazaki K. A comparative study between flow visualization and computational fluid dynamic analysis for the Sun Medical centrifugal blood pump. Artif Organs. 2004;28: 458-66.

32. Biancucci BA, Deutsch S, Geselowitz DB, Tarbell JM. In vitro studies of gas bubble formation by mechanical heart valves. J Heart Valve Dis. 1999;8:186-96.

33. Burgreen GW, Antaki JF, Griffith BP. A design improvement strategy for axial blood pumps using computational fluid dynamics. ASAIO J. 1996;42:M354-60.

34. Sukumar R, Athavale MM, Makhijani VB, Przekwas AJ. Application of computational fluid dynamics techniques to blood pumps. Artif Organs. 1996;20:529-33.

35. Song X, Throckmorton AL, Wood HG, Allaire PE, Olsen DB. Transient and quasi-steady computational fluid dynamics study of a left ventricular assist device. ASAIO J. 2004;50:410-7. 